\title{
Improving upper bounds for the distinguishing index
}

\author{
Monika Pilśniak* \\ AGH University, Department of Discrete Mathematics, \\ al. Mickiewicza 30, 30-059 Krakow, Poland
}

Received 27 November 2015, accepted 13 February 2017, published online 6 March 2017

\begin{abstract}
The distinguishing index of a graph $G$, denoted by $D^{\prime}(G)$, is the least number of colours in an edge colouring of $G$ not preserved by any non-trivial automorphism. We characterize all connected graphs $G$ with $D^{\prime}(G) \geq \Delta(G)$. We show that $D^{\prime}(G) \leq 2$ if $G$ is a traceable graph of order at least seven, and $D^{\prime}(G) \leq 3$ if $G$ is either claw-free or 3-connected and planar. We also investigate the Nordhaus-Gaddum type relation: $2 \leq D^{\prime}(G)+D^{\prime}(\bar{G}) \leq$ $\max \{\Delta(G), \Delta(\bar{G})\}+2$ and we confirm it for some classes of graphs.
\end{abstract}

Keywords: Edge colouring, symmetry breaking in graph, distinguishing index, claw-free graph, planar graph.

Math. Subj. Class.: 05C05, 05C10, 05C15, 05C45

\section{Introduction}

We follow standard terminology and notation of graph theory (cf. [12]). In this paper, we consider general, i.e. not necessarily proper, edge colourings of graphs. Such a colouring $f$ of a graph $G$ breaks an automorphism $\varphi \in \operatorname{Aut}(G)$ if $\varphi$ does not preserve colours of $f$. The distinguishing index $D^{\prime}(G)$ of a graph $G$ is the least number $d$ such that $G$ admits an edge colouring with $d$ colours that breaks all non-trivial automorphisms (such a colouring is called a distinguishing edge d-colouring). Clearly, $D^{\prime}\left(K_{2}\right)$ is not defined, so in this paper, a graph $G$ is called admissible if neither $G$ nor $\bar{G}$ contains $K_{2}$ as a connected component.

The definition of $D^{\prime}(G)$ introduced by Kalinowski and Pilśniak in [17] was inspired by the distinguishing number $D(G)$ which was defined for general vertex colourings by Albertson and Collins [1]. Another concept is the distinguishing chromatic number $\chi_{D}(G)$

\footnotetext{
* The research was partially supported by the Polish Ministry of Science and Higher Education. The author would like to thank an anonymous reviewer whose remarks and comments have been a great help in preparing the final version.

E-mail address: pilsniak@agh.edu.pl (Monika Pilśniak)
}

(a) (i) This work is licensed under http://creativecommons.org/licenses/by/3.0/ 
introduced by Collins and Trenk [7] for proper vertex colourings. Both numbers, $D(G)$ and $\chi_{D}(G)$, have been intensively investigated by many authors in recent years $[4,5,6,9,16]$.

Our investigation was motivated by the renowned result of Nordhaus-Gaddum [18] who proved in 1956 the following lower and upper bounds for the sum of the chromatic numbers of a graph and its complement (actually, the upper bound was first proved by Zykov [22] in 1949).

Theorem 1.1 ([18]). If $G$ is a graph of order $n$ with the chromatic number $\chi(G)$, then

$$
2 \sqrt{n} \leq \chi(G)+\chi(\bar{G}) \leq n+1 .
$$

Since then, Nordhaus-Gaddum type bounds were obtained for many graph invariants. An exhaustive survey is given in [2]. Here, we adduce only those closely related to the topic of our paper.

In 1964, Vizing [20] considered proper edge colourings and he proved NordhausGaddum type bounds for the chromatic index of a graph.

Theorem 1.2 ([20]). If $G$ is a graph of order $n$ with the chromatic index $\chi^{\prime}(G)$, then

$$
n-1 \leq \chi^{\prime}(G)+\chi^{\prime}(\bar{G}) \leq 2(n-1)
$$

In 2013, Collins and Trenk [8] proved Nordhaus-Gaddum type inequalities for the distinguishing chromatic number.

Theorem 1.3 ([8]). For every graph of order $n$ and distinguishing number $D(G)$ the following inequalities are satisfied

$$
2 \sqrt{n} \leq \chi_{D}(G)+\chi_{D}(\bar{G}) \leq n+D(G) .
$$

Kalinowski and Pilśniak [17] also introduced a distinguishing chromatic index $\chi_{D}^{\prime}(G)$ of a graph $G$ as the least number of colours in a proper edge colouring that breaks all non-trivial automorphisms of $G$. They proved the following somewhat unexpected result.

Theorem 1.4 ([17]). If $G$ is a connected graph of order $n \geq 3$, then

$$
\chi_{D}^{\prime}(G) \leq \Delta(G)+1
$$

unless $G \in\left\{C_{4}, K_{4}, C_{6}, K_{3,3}\right\}$ when $\chi_{D}^{\prime}(G) \leq \Delta(G)+2$.

The following Nordhaus-Gaddum type inequalities for the distinguishing chromatic index are the same as in Theorem 1.2 but we have to be more careful in the proof.

Theorem 1.5. If $G$ is an admissible graph of order $n \geq 3$, then

$$
n-1 \leq \chi_{D}^{\prime}(G)+\chi_{D}^{\prime}(\bar{G}) \leq 2(n-1)
$$

with the only exception $K_{1,4}$.

Proof. Without loss of generality we may assume that $G$ is connected. It can be easily checked that the conclusion holds if $G \in\left\{K_{4}, C_{6}, \overline{C_{6}}, K_{3,3}\right\}$. Otherwise, $\chi_{D}^{\prime}(G) \leq$ $\Delta(G)+1$. Suppose first that $\bar{G}$ is also connected. By Theorem 1.4,

$$
\Delta(G)+\Delta(\bar{G}) \leq \chi_{D}^{\prime}(G)+\chi_{D}^{\prime}(\bar{G}) \leq \Delta(G)+\Delta(\bar{G})+2 .
$$


Clearly, $n-1 \leq \Delta(G)+\Delta(\bar{G}) \leq 2(n-2)$ since both $G$ and $\bar{G}$ are connected.

Now, let $\bar{G}$ be disconnected (but admissible). If there are two nonisomorphic components of $\bar{G}$ of orders $k_{1}$ and $k_{2}$ such that $3 \leq k_{1} \leq k_{2}$, then $\Delta(\bar{G}) \leq n-k_{1}-1 \leq n-4$, so $\chi_{D}^{\prime}(\bar{G}) \leq n-2$. If $\bar{G}$ has $t \geq 2$ components isomorphic to a graph $H$ of order at least three, then $\chi_{D}^{\prime}(H) \leq \frac{n}{t}+1$ as $\Delta(H) \leq \frac{n}{t}-1$. Even if we wastefully add an extra colour for each additional copy of $H$, we get $\chi_{D}^{\prime}(t H) \leq \frac{n}{t}+1+t-1=\frac{n}{t}+t \leq n-2$ unless $G=K_{3,3}$ but this we already checked.

To complete the proof it is enough to settle the case when $\bar{G}$ has only one component $H$ of order at least three and some isolated vertices. Hence, $\Delta(H) \leq n-2$. It is easy to check that $\chi_{D}^{\prime}(G)+\chi_{D}^{\prime}(\bar{G}) \leq 2(n-1)$ for $H \in\left\{K_{4}, C_{6}, \overline{C_{6}}, K_{3,3}\right\}$ except for $H=K_{4}$ when $G=K_{1,4}$. Otherwise, $\chi_{D}^{\prime}(\bar{G}) \leq n-1$ and the conclusion holds unless $|G|=|H|+1$ and $\Delta(H)=n-2$. But then $G$ has a unique vertex $x$ of degree $n-1$ (hence, $x$ is fixed by every automorphism of $G$ ) with a pendant edge. The graph $G-x$ has a distinguishing colouring with $n-1$ colours by Theorem 1.4 since $\Delta(G-x) \leq n-2$. It suffices to colour the pendant edge with a colour missing at $x$ to see that $\chi_{D}^{\prime}(G) \leq n-1$.

Collins and Trenk observed in [8] that the Nordhaus-Gaddum type relation is trivial for the distinguishing number, as $D(G)+D(\bar{G})=2 D(G)$ since $\operatorname{Aut}(\bar{G})=\operatorname{Aut}(G)$ and every colouring of $V(G)$ breaking all non-trivial automorphisms of $G$ also breaks those of $\bar{G}$.

In Section 4 we formulate and discuss the following conjecture.

Conjecture 1.6. Let $G$ be an admissible graph of order $n \geq 7$, and let $\Delta=\max \{\Delta(G)$, $\Delta(\bar{G})\}$. Then

$$
2 \leq D^{\prime}(G)+D^{\prime}(\bar{G}) \leq \Delta+2 .
$$

In Section 2 we characterize graphs $G$ which need exactly $\Delta(G)$ colours to break all non-trivial automorphisms. In Section 3 we give upper bounds for the distinguishing index of traceable graphs, claw-free graphs, planar graphs and 2-connected graphs.

\section{Improved general upper bound}

In the sequel, we make use of some facts proved in [17].

Proposition 2.1 ([17]). $D^{\prime}\left(P_{n}\right)=2$ for every $n \geq 3$.

Proposition 2.2 ([17]). $D^{\prime}\left(C_{n}\right)=3$ for $n \leq 5$, and $D^{\prime}\left(C_{n}\right)=2$ for $n \geq 6$.

Proposition 2.3 ([17]). $D^{\prime}\left(K_{n}\right)=3$ if $3 \leq n \leq 5$, and $D^{\prime}\left(K_{n}\right)=2$ if $n \geq 6$.

Proposition 2.4 ([17]). $D^{\prime}\left(K_{3,3}\right)=3$, and $D^{\prime}\left(K_{n, n}\right)=2$ if $n \geq 4$.

By the well-known theorem of Jordan (cf. [12]), every finite tree $T$ has either a central vertex or a central edge, which is fixed by every automorphism of $T$. In the proof of Theorem 2.8, which is the main result of this section, we use Lemma 2.5, a simple generalization of the theorem of Jordan. Recall that the eccentricity of a vertex $v$ in a connected graph $G$ is the number

$$
\varepsilon_{G}(v)=\max \{d(v, u): u \in V(G)\} .
$$

The center of a graph $G$ is the set $Z(G)$ of vertices with minimum eccentricity. Clearly, the center of $G$ is setwise fixed by every automorphism $\varphi \in \operatorname{Aut}(G)$, i.e. $\varphi(v) \in Z(G)$ if $v \in Z(G)$. A proper subgraph $H$ of $G$ is called pendant if it has only one vertex adjacent to vertices outside $H$. 
Lemma 2.5. Let $G$ be a connected graph such that every cycle is contained in a clique. Then the center of $G$ is either a single vertex or a maximal clique.

Proof. The claim is true if $G$ is a clique $K_{k}$ of order $k \geq 1$. Otherwise, $\kappa(G)=1$, and each block of $G$ is a clique of order at least two. We then modify the standard proof of the theorem of Jordan for trees. Let $G^{-}$be a graph obtained from $G$ by deleting $k-1$ vertices of degree $k-1$ in every pendant clique $K_{k}$ with $k \geq 2$. Clearly, $\varepsilon_{G^{-}}(v)=\varepsilon_{G}(v)-1$ for each $v \in V\left(G^{-}\right)$. Consequently, $Z\left(G^{-}\right)=Z(G)$. We continue this process until only one clique $K_{k}$ is left for some $k \geq 1$. This clique is maximal whenever $k \geq 2$.

A symmetric tree, denoted by $T_{h, d}$, is a tree with a central vertex $v_{0}$, all leaves at the same distance $h$ from $v_{0}$ and all vertices that are not leaves of equal degree $d$. A bisymmetric tree, denoted by $T_{h, d}^{\prime \prime}$, is a tree with a central edge $e_{0}$, all leaves at the same distance $h$ from the edge $e_{0}$ and all vertices which are not leaves of equal degree $d$.

Theorem 2.6 ([17]). If $T$ is a tree of order $n \geq 3$, then $D^{\prime}(T) \leq \Delta(T)$. Moreover, equality is achieved if and only if $T$ is either a symmetric or a bisymmetric tree.

For connected graphs in general there is the following upper bound for $D^{\prime}(G)$.

Theorem 2.7 ([17]). If $G$ is a connected graph of order $n \geq 3$, then

$$
D^{\prime}(G) \leq \Delta(G)
$$

unless $G$ is $C_{3}, C_{4}$ or $C_{5}$.

It follows for connected graphs that $D^{\prime}(G)>\Delta(G)$ if and only if $D^{\prime}(G)=\Delta(G)+1$ and $G$ is a cycle of length at most 5. The equality $D^{\prime}(G)=\Delta(G)$ holds for cycles of length at least 6 , for $K_{4}, K_{3,3}$ and for all symmetric or bisymmetric trees. Now, we show that $D^{\prime}(G)<\Delta(G)$ for all other connected graphs. A palette of a vertex is the multiset of colours of edges incident to it.

Theorem 2.8. Let $G$ be a connected graph that is neither a symmetric nor a bisymmetric tree. If the maximum degree of $G$ is at least 3 , then

$$
D^{\prime}(G) \leq \Delta(G)-1
$$

unless $G$ is $K_{4}$ or $K_{3,3}$.

Proof. Denote $\Delta=\Delta(G)$. The conclusion holds for trees due to Theorem 2.6. Then assume that $G$ contains a cycle. The general idea of the proof is the following. If $G$ does not contain a cycle of length greater than three, then we define $G^{\prime}$ as an empty graph. Otherwise, we consecutively delete pendant trees and pendant triangles until we obtain a subgraph $G^{\prime}$. Then, we construct an edge colouring $f$ with $\Delta-1$ colours stabilizing all vertices of $G^{\prime}$ by every automorphism preserving $f$. Finally, we colour pendant subtrees and pendant triangles to complete a distinguishing colouring with $\Delta-1$ colours of the whole graph $G$.

If $\Delta\left(G^{\prime}\right)=2$, then $G^{\prime}$ is a cycle $C_{p}$ having a distinguishing colouring with $\Delta-1$ colours unless $p \in\{4,5\}$ and $\Delta=3$. In this case, it can be easily checked that the graph $G_{+}^{\prime}$ induced by $C_{p}$ and the independent edges of $G$ incident to $C_{p}$ can always be coloured with two colours such that the vertices of $C_{p}$ are fixed by every colour preserving 
automorphism. So we can assume that $\Delta\left(G^{\prime}\right) \geq 3$. If $G^{\prime} \in\left\{K_{4}, K_{3,3}\right\}$, then $G^{\prime} \neq G$ due to the assumption, hence $\Delta \geq 4$, so we can stabilize $K_{4}$ or $K_{3,3}$ with three colours.

Let $N_{i}(v)$ denote the $i$-th sphere in $v$, i.e. the set of vertices of distance $i$ from the vertex $v$. Let $x$ be a vertex with maximum degree in $G^{\prime}$. We colour with 1 all edges incident with $x$. In our edge colouring $f$ of the graph $G^{\prime}$, the vertex $x$ will be the unique vertex of maximum degree with the monochromatic palette $\{1, \ldots, 1\}$. Hence, $x$ will be fixed by every automorphism $\varphi$ preserving $f$. Consequently, $\varphi$ maps each sphere $N_{i}(x)$ onto itself.

The first sphere $N_{1}(x)$ can be partitioned into subsets $M_{k}$, for $k=0, \ldots, \Delta-1$, defined as

$$
M_{k}=\left\{v \in N_{1}(x):\left|N_{1}(v) \cap N_{2}(x)\right|=k\right\} .
$$

Denote $M_{k}=\left\{v_{1}, \ldots, v_{l_{k}}\right\}$. Thus, $l_{0}+l_{1}+\ldots+l_{\Delta-1}=\Delta$.

We want to find a colouring $f$ of the edges of $G^{\prime}\left[N_{1}(x) \cup N_{2}(x)\right]$ and, if necessary, of some subsequent spheres, such that each vertex of $N_{1}(x) \cup N_{2}(x)$ is fixed by every automorphism preserving this colouring. To do this, we proceed in a number of steps $M_{k}$, for $k=0, \ldots, \Delta-1$. In each step $M_{k}$, we find a colouring that fixes the vertices of $M_{k}$ and their neighbours in $N_{2}(x)$.

Step $\mathbf{M}_{0}$. First we consider the case when the subgraph $G^{\prime}\left[M_{0}\right]$ induced by the vertices of $M_{0}$ is connected. Observe that $\Delta\left(G^{\prime}\left[M_{0}\right]\right) \leq \Delta-1$ and, by Theorem 2.7, we can colour distinguishingly the edges of $G^{\prime}\left[M_{0}\right]$ with $\Delta-1$ colours, even if $G^{\prime}\left[M_{0}\right]$ is a short cycle $C_{p}$ with $3 \leq p \leq 5$. Indeed, if $G^{\prime}\left[M_{0}\right]=C_{3}$ and $\Delta=3$, then we would have $G=K_{4}$, but $K_{4}$ is excluded. Otherwise, $\Delta \geq 4$ and we can use a third colour in a short cycle $C_{p}$. It may happen that there exists a vertex $v \in M_{0}$ of degree $\Delta$ in $G^{\prime}$ (so $\left|M_{0}\right|=\Delta$ ) with a monochromatic palette $\{1, \ldots, 1\}$ in a colouring of $G^{\prime}\left[M_{0}\right]$ given by Theorem 2.7. In this case, either $G$ is a complete graph $K_{n}$ with $n \geq 5$ so $D^{\prime}\left(K_{n}\right) \leq \Delta-1$ by Proposition 2.3 , or it is not difficult to see that there exists a colour $c$ such that there is no vertex with all incident edges coloured with $c$; whence we can exchange $c$ and 1 in this colouring of $G^{\prime}\left[M_{0}\right]$.

Now, let $G^{\prime}\left[M_{0}\right]$ be disconnected. Let $z_{1}, \ldots, z_{s}$ be isolated vertices or end-vertices of isolated edges in $G^{\prime}\left[M_{0}\right]$. Clearly, $s \leq \Delta-1$ by the definition of $G^{\prime}$. If $s=\Delta-1$, then we colour with $i$ every edge $z_{i} u$, where $u \in N_{1}(x) \backslash M_{0}$. Otherwise, we colour $z_{i} u$ with $i+1$ for $i=1, \ldots, s$. Thus, we avoid a monochromatic palette of $\{1, \ldots, 1\}$ at another vertex of maximum degree in $G^{\prime}$.

We also have to distinguish all isomorphic components of $G^{\prime}\left[M_{0}\right]$ of order greater than 2. Denote such a component by $H$ and suppose that $G^{\prime}\left[M_{0}\right]$ contains $t$ components isomorphic to $H$, for some $t \geq 2$. Hence $t \leq \frac{\Delta}{3}$ and $\Delta(H) \leq \frac{\Delta}{t}-1$. Therefore, we can choose distinct sets of $\frac{\Delta}{t}$ colours for every component since

$$
\left(\begin{array}{c}
\Delta-1 \\
\frac{\Delta}{t}
\end{array}\right) \geq\left(\begin{array}{c}
\Delta-1 \\
3
\end{array}\right) \geq \frac{\Delta}{3} \geq t .
$$

Thus each vertex of $M_{0}$ is fixed.

Step $\mathbf{M}_{\mathbf{1}}$. For every $i=1, \ldots, l_{1}$, we colour the edge $v_{i} u$, where $u \in N_{2}(x)$, with a distinct colour from $\{1, \ldots, \Delta-1\}$. This is impossible only if $l_{1}=\Delta$, when we have to have two vertices $a, b \in M_{1}$ with the same colour of edges $a a^{\prime}$ and $b b^{\prime}$, where $a^{\prime}$ and $b^{\prime}$ are neighbours of $a$ and $b$ in $N_{2}(x)$, respectively. If $G^{\prime}\left[M_{1}\right]$ contains an edge $e$, then we colour it with 1 , and all other edges of $G^{\prime}\left[M_{1}\right]$ with 2 . Then we choose exactly one of the vertices $a, b$ incident to $e$. We proceed analogously when $G^{\prime}\left[N_{2}(x)\right]$ contains an edge. Then all 
vertices of $M_{1}$ are fixed unless $l_{1}=\Delta$ and neither $G^{\prime}\left[N_{1}(x)\right]$ nor $G^{\prime}\left[N_{2}(x)\right]$ contains an edge.

If $\left|N_{2}(x)\right|=1$, then $G^{\prime}$ is isomorphic to $K_{2, \Delta}$. It is easy to see that $D^{\prime}\left(K_{2, \Delta}\right) \leq \Delta-1$ for $\Delta \geq 3$ (for $\Delta \geq 4$ this immediately follows from Lemma 3.1 and Corollary 3.8). If $2 \leq\left|N_{2}(x)\right| \leq \Delta-1$, then choosing $a$ and $b$ such that $a^{\prime}$ has at least two neighbours in $N_{1}(x)$ and $b^{\prime} \neq a^{\prime}$ yields a colouring fixing $N_{1}(x) \cup N_{2}(x)$.

Suppose $\left|N_{2}(x)\right|=\Delta$. If there is a vertex $v \in N_{2}(x)$ with less than $\Delta-1$ neighbours in $N_{3}(x)$, then we choose $a$ such that $a^{\prime}=v$, and it suffices to reserve a unique set of colours for the edges between $a^{\prime}$ and $N_{3}(x)$.

Hence, assume that every vertex of $N_{2}(x)$ has $\Delta-1$ neighbours in $N_{3}(x)$. We select two vertices $a, b \in M_{1}$ and assume that the colours of the edges $a a^{\prime}$ and $b b^{\prime}$ are the same. Next, we implement the following Procedure SUBTREES $(a, b)$, which we also use in subsequent steps.

Procedure SUBTREES $(a, b)$

We are given two vertices $a, b \in N_{1}(x)$ such that each their neighbour in $N_{2}(x)$ is adjacent to $\Delta-1$ vertices of $N_{3}(x)$.

Let $T_{a}$ be a maximal subtree of the graph $G^{\prime}\left[\{a\} \cup \bigcup_{i \geq 2} N_{i}(x)\right]$, rooted at $a$, such that all leaves of $T_{a}$ belong to the same sphere $N_{l-1}(x)$ and each vertex of $V\left(T_{a}\right) \cap N_{i-1}(x)$ has $\Delta-1$ neighbours in $N_{i}(x)$ for $i=3, \ldots, l$. Thus $l \geq 3$. Define a graph

$$
\widetilde{T_{a}}=G^{\prime}\left[\bigcup_{v \in V\left(T_{a}\right) \backslash\{a\}} N(v)\right],
$$

i.e. $\widetilde{T}_{a}$ is a graph obtained from $T_{a}$ by adding all edges incident with the leaves of $T_{a}$. Analogously, we define a tree $T_{b}$ and a graph $\widetilde{T}_{b}$. Observe that the trees $T_{a}$ and $T_{b}$ are disjoint and non-empty.

The edges incident to the roots $a$ and $b$ are already coloured. For every other vertex of $T_{a}$ and $T_{b}$, we colour its incident edges going to the next sphere with distinct colours from $\{1, \ldots, \Delta-1\}$. Thus we obtain an edge colouring $f$. The only automorphism of $T_{a}$ (as well as of $T_{b}$ ) preserving $f$ is the identity. The vertex $x$ will be fixed by every colour preserving automorphism $\varphi$. Consequently, $\varphi$ maps $\widetilde{T}_{a}$ onto $\widetilde{T}_{b}$ whenever $\varphi(a)=b$. Thus, if $\widetilde{T}_{a}$ and $\widetilde{T}_{b}$ are not isomorphic, then $f$ distinguishes all vertices in $V\left(T_{a}\right) \cup V\left(T_{b}\right)$. Hence, assume that the rooted graphs $\widetilde{T}_{a}$ and $\widetilde{T}_{b}$ are isomorphic. Observe that there exists exactly one non-trivial isomorphism $\psi_{0}: V\left(T_{a}\right) \rightarrow V\left(T_{b}\right)$ preserving $f$ since each vertex in $T_{a}$ has a distinct coloured path from the root $a$.

Denote $W_{l}=\left(V\left(\widetilde{T}_{a}\right) \cup V\left(\widetilde{T}_{b}\right)\right) \cap N_{l}(x)$. By our choice of $G^{\prime}$, all vertices in $W_{l}$ are of degree at least two in $G^{\prime}$. It follows that one of the following three cases has to hold.

Case 1. There exist vertices in $W_{l}$ adjacent to more than one vertex of $W_{l-1}$. Then we modify $f$ by colouring again all edges between such vertices and $W_{l-1}$ in order to break any possible permutation of $W_{l}$. A permutation of a set $L \subseteq W_{l}$ can be extended to an automorphism of $G^{\prime}$ that fixes all leaves of $\widetilde{T}_{a} \cup \widetilde{T}_{b}$ only if every vertex from $L$ have the same set of neighbours $U=\left\{u_{1}, \ldots, u_{d}\right\}$ in $W_{l-1}$. Such a set $L$ contains at most $\Delta-1$ leaves since the number of edges joining $U$ to $W_{l}$ equals $d(\Delta-1)$. Every permutation of $L$ will be broken whenever for every vertex $w \in L$ the multiset of colours of the edges $w u_{1}, \ldots, w u_{d}$ will be distinct. Clearly, $d \leq \Delta$. There are $\left(\begin{array}{c}\Delta+d-2 \\ d\end{array}\right)$ such possible multisets of $\Delta-1$ colours. Clearly, $\left(\begin{array}{c}\Delta+d-2 \\ d\end{array}\right)-1 \geq \Delta-1$ for $\Delta \geq 3$ and $d \geq 2$. We can exclude a 


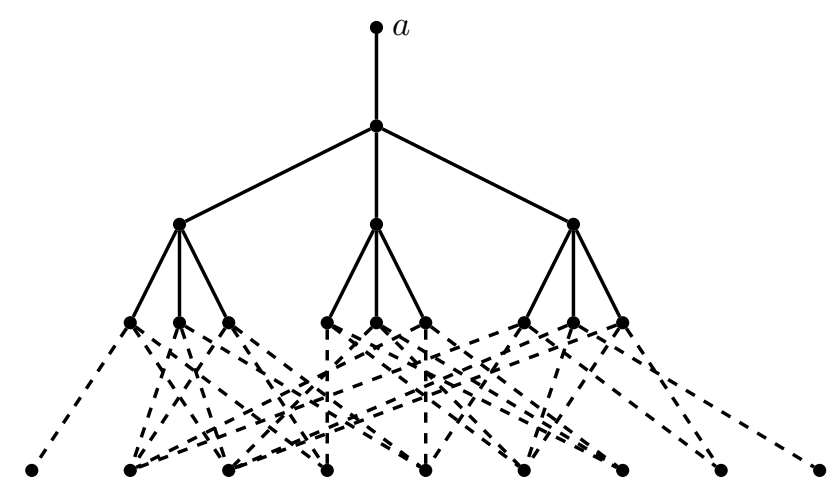

Figure 1: An example of the subgraph $\widetilde{T}_{a}$ for $\Delta=4$ and $l=4$. The edges of $\widetilde{T}_{a}$ between $W_{3}$ and $W_{4}$ that do not belong to the tree $T_{a}$ are dashed.

rainbow multiset $P=\{1, \ldots, d\}$ (or an almost rainbow multiset $P=\{1, \ldots, \Delta-1, \Delta-1\}$ if $d=\Delta$ ) and we still have enough multisets to colour the edges incident with vertices of $L$. Moreover, for $d=\Delta$ we can also exclude a monochromatic palette $\{1, \ldots, 1\}$ since $\left(\begin{array}{c}2 \Delta-2 \\ \Delta\end{array}\right)-2 \geq \Delta-1$ for $\Delta \geq 3$.

We partition the set $W_{l}$ into maximal subsets $L$ with the same set of neighbours and assign suitable multisets of colours to each set $L$. We thus obtain a colouring fixing all vertices from $W_{l}$ unless $\psi_{0}$ can be extended to an isomorphism $\widetilde{\psi_{0}}$ of $\widetilde{T}_{a}$ onto $\widetilde{T}_{b}$ preserving this colouring. To break every such possible extension $\widetilde{\psi_{0}}$, it suffices to assign the excluded multiset $P$ to one vertex of one set $L$.

Case 2. Every vertex in $W_{l}$ has only one neighbour in $W_{l-1}$ and the set of edges $F=E\left(G^{\prime}\left[W_{l}\right]\right)$ is non-empty. Then we colour one edge of $F$ with 1 , and all other edges in $F$ with 2. This colouring fixes all vertices of $\widetilde{T}_{a}$ and $\widetilde{T}_{a}$ unless all edges in $F$ are of the form $w \widetilde{\psi_{0}}(w)$, where $w \widetilde{\psi_{0}}(w)$ is one of possible extensions of $\psi_{0}$ to an isomorphism of $\widetilde{T}_{a}$ onto $\widetilde{T}_{b}$. In such a case, we choose one edge $w w^{\prime} \in F$ and exchange colours of the edge $w u$, where $u \in W_{l-1}$, with another edge between $u$ and $W_{l}$.

Case 3. Every vertex in $W_{l}$ has only one neighbour in $W_{l-1}$ and no neighbours in $W_{l}$. By the maximality of the trees $T_{a}$ and $T_{b}$ and the definition of $G^{\prime}$, each vertex in $W_{l}$ has at least one neighbour in $N_{l+1}(x)$ and there exists a vertex $w_{0} \in W_{l}$ with $s<$ $\Delta-1$ neighbours $y_{1}, \ldots, y_{s} \in N_{l+1}(x)$. We colour each edge $w_{0} y_{j}$ with colour $j+1$ for $j=1, \ldots, s$. Next, for every vertex $w \in W_{l}$, we colour the set of edges between $w$ and $N_{l+1}(x)$ with a set of $\Delta-1$ colours excluding the set $\{2, \ldots, s+1\}$.

We thus obtained a colouring $f$ of the edges of $G^{\prime}\left[V\left(\widetilde{T}_{a}\right) \cup V\left(\widetilde{T}_{b}\right)\right]$, and the edges incident to $W_{l}$ in Case 3 , fixing all vertices of $\widetilde{T}_{a}$ and $\widetilde{T}_{b}$.

End of Procedure SUBTREES $(a, b)$

Step $\mathbf{M}_{\mathbf{2}}$. For every $i=1, \ldots, l_{2}$, we colour the edges $v_{i} u_{i}^{1}, v_{i} u_{i}^{2}$ where $\left\{u_{i}^{1}, u_{i}^{2}\right\} \subseteq$ $N_{2}(x)$, with distinct sets of colours from among $\left(\begin{array}{c}\Delta-1 \\ 2\end{array}\right)$ sets. This is impossible only in the following three cases (in each case, we can assume that neither $G^{\prime}\left[N_{1}(x)\right]$ nor $G^{\prime}\left[N_{2}(x)\right]$ contains an edge, otherwise we could construct a distinguishing colouring $f$ of $G^{\prime}\left[N_{1}(x) \cup\right.$ $N_{2}(x)$ ] analogously as in step $M_{1}$ ): 
a) $l_{2}=\Delta=4$. If there exist two vertices $a$ and $b$ in $M_{2}$ such that $N(a) \cap N(b) \cap$ $N_{2}(x) \neq \emptyset$, then we colour with 2 both edges incident with $b$, and for the remaining vertices in $M_{2}$ we have distinct sets of colours from among $\left(\begin{array}{l}3 \\ 2\end{array}\right)$ sets. If for every two vertices $a, b \in M_{2}$, the set $N(a) \cap N(b) \cap N_{2}(x)$ is empty, then two vertices $a$ and $b$ are assign the same pair of distinct colours, and we can distinguish them in next spheres using the procedure SUBTREES $(a, b)$.

b) $l_{2}=\Delta-1$ and $\Delta=3$. Let $M_{2}=\{a, b\}$. If $N(a) \cap N(b) \cap N_{2}(x) \neq \emptyset$, then we colour edges incident with $a$ with colours 1 and 2 , and both edges incident with $b$ with 2. If the set $N(a) \cap N(b) \cap N_{2}(x)$ is empty, then $a$ and $b$ get the same pair of distinct colours and we can distinguish them in next spheres by the procedure SUBTREES $(a, b)$.

c) $l_{2}=\Delta=3$. Let $M_{2}=\{a, b, c\}$. If for two vertices of $M_{2}$, say $a$ and $b$, the set $N(a) \cap N(b) \cap N_{2}(x)$ is non-empty, then we can colour with 2 both edges incident with $b$ and we colour edges incident with the remaining vertices of $M_{2}$ with a couple $\{1,2\}$. It is not difficult to verify that this way, for every configuration of neighbours of $M_{2}$, we can obtain colouring fixing the vertices of $N_{1}(x) \cup N_{2}(x)$ unless $\mid N(a) \cap$ $N(b) \cap N(c) \cap N_{2}(x) \mid=2$. But then $G^{\prime}=G=K_{3,3}$, contrary to the assumption. If every vertex of $N_{2}(x)$ is adjacent only to one vertex of $M_{2}$, then the pairs of edges incident to $a$ and $b$ are assign the same pair of colours $\{1,2\}$, and we distinguish them using the procedure SUBTREES $(a, b)$. Both edges $c u^{1}, c u^{2}$ incident with $c$ are coloured with 2 , and to distinguish them, we split $c$ into two vertices $c^{1}$ and $c^{2}$, each joined by an edge coloured with 2 to $u^{1}$ and $u^{2}$, respectively, and apply the procedure SUBTREES $\left(c^{1}, c^{2}\right)$.

Step $\mathbf{M}_{\mathbf{k}}$, for $\mathbf{k} \geq \mathbf{3}$. For every $i=1, \ldots, l_{k}$, we colour the edges between $v_{i}$ and $N_{2}(x)$ with distinct sets of $k$ colours from among $\left(\begin{array}{c}\Delta-1 \\ k\end{array}\right)$ sets. It is always possible whenever $\left(\begin{array}{c}\Delta-1 \\ k\end{array}\right) \geq l_{k}$. This inequality does not hold only in two cases:

a) $k=\Delta-2$ and $l_{k}=\Delta$. In this case we define a colouring with $\Delta-1$ colours like in step $M_{2}$ a). Namely, if either a vertex of $M_{k}$ or its neighbour in $N_{2}(x)$ is adjacent to a vertex in the same sphere, then we can define a colouring fixing all these vertices analogously as in step $M_{1}$ and step $M_{2}$. Also, if there are two vertices $a, b \in M_{\Delta-2}$ with a common neighbour in $N_{2}(x)$, we can assign the same palette to $a$ and $b$ as in the previous steps. Otherwise, two vertices $a, b \in M_{\Delta-2}$ are assign the same palette of $\Delta-2$ colours and we distinguish them using Procedure SUBTREES $(a, b)$.

b) $k=\Delta-1$ and $l_{k} \geq 2$. Hence, $\Delta \geq 4$. For every $i=1, \ldots, l_{k}$, the set of edges between $v_{i} \in M_{\Delta-1}$ and $N_{2}(x)$ will be assign a distinct multiset $P^{i}$ of colours from the set $\{1, \ldots, \Delta-1\}$, where only colour $i$ appears twice. Moreover, one vertex can assign a rainbow palette $\{1, \ldots, \Delta-1\}$. Thus every vertex of $M_{\Delta-1}$ will have a distinct palette, and hence will be stabilized. To stabilize the two vertices of $N_{2}(x)$ joined to $v_{i}$ by edges of colour $i$, we examine the vertices $v_{1}, \ldots, v_{\Delta-1}$ of $M_{\Delta-1}$ in the following order.

First, we consider each vertex $v_{i}$ that have a neighbour $w_{i} \in N_{2}(x)$ with at least one but at most $\Delta-2$ neighbours in $N_{3}(x)$. We choose another neighbour $w_{i}^{\prime} \in N_{2}(x)$ of $v_{i}$ and assign two distinct sets of colours for the edges going to $N_{3}(x)$ from $w_{i}$ and $w_{i}^{\prime}$, respectively. We colour the edges $v_{i} w_{i}$ and $v_{i} w_{i}^{\prime}$ with the same colour $i$. Thus all neighbours of $v_{i}$ are stabilized. 
In the next stage, we consider every vertex $v_{i}$ with every neighbour in $N_{2}(x)$ adjacent to $\Delta-1$ vertices of $N_{3}(x)$. We colour the set of edges between $v_{i}$ and $N_{2}(x)$ with the palette $P^{i}$, where two edges $v_{i} u_{1}, v_{i} u_{2}$ are coloured with $i$. Then we delete $v_{i}$ and introduce two vertices $v_{i}^{1}, v_{i}^{2}$ and edges $v_{i}^{1} u_{1}$ and $v_{i}^{2} u_{2}$ coloured with $i$. Then we use the procedure SUBTREES $\left(v_{i}^{1}, v_{i}^{2}\right)$ to stabilize $u_{1}$ and $u_{2}$.

Further, we consider each vertex $v_{i}$ with a neighbour $w_{i} \in N_{2}(x)$ incident to an edge $w_{i} u$, where $u \in N_{2}(x)$. First, we look for such an edge $w_{i} u$, which is already coloured. If there is no such edge, we take an uncoloured $w_{i} u$ and colour it with colour 3 . In both cases, we put colour $i$ on the edge $v_{i} w_{i}$ and another edge $v_{i} w$ with $w \neq u$. After we examine each such vertex $v_{i}$, we colour with 2 all remaining edges contained in $\mathrm{N}_{2}(x)$.

Finally, we are left with at most $\Delta$ vertices $v_{i}$ such that every neighbour of $v_{i}$ is adjacent only to (at least two) vertices of $N_{1}(x)$. We take a first such vertex $v_{i}$ and assign colour $i$ to two its incident edges $v_{i} w_{i}$ and $v_{i} w_{i}^{\prime}$. Thus all neighbours of $v_{i}$ are stabilized unless common neighbours of $w_{i}$ and $w_{i}^{\prime}$ were not considered yet. Then we take such a neighbour $v_{j}$ and colour its incident edges with the palette $P^{j}$ such that the edges $v_{j} w_{i}$ and $v_{j} w_{i}^{\prime}$ have distinct colours. We repeat this procedure until only one vertex of $M_{\Delta-1}$ is left. We put a rainbow palette $\{1, \ldots, \Delta-1\}$ on its incident edges.

After we accomplish steps $M_{0}, \ldots, M_{\Delta-1}$, we colour all uncoloured edges in subgraphs $G^{\prime}\left[N_{1}(x)\right]$ and $G^{\prime}\left[N_{2}(x)\right]$ with 2. Each vertex of $N_{1}(x) \cup N_{2}(x)$ is now fixed by every automorphism preserving our colouring $f$ of edges of $G^{\prime}\left[\{x\} \cup N_{1}(x) \cup N_{2}(x)\right]$, and of some edges between next spheres, if the procedure SUBTREES was used.

Then we recursively colour all yet uncoloured edges incident to consecutive spheres $N_{i}(x)$ as follows: for $v \in N_{i}(x), i \geq 2$, we colour all edges $v u$, where $u \in N_{i+1}(x)$, with distinct colours from $\{1, \ldots, \Delta-1\}$. This is always possible since every vertex of $N_{i}(x)$ has at most $\Delta-1$ neighbours in $N_{i+1}(x)$. Finally, we colour all uncoloured edges with end-vertices in the same sphere with 2 . Hence, all vertices of $G^{\prime}$ are fixed by any automorphism preserving our colouring $f$. It is also easily seen that the already coloured edges can save their colours. Moreover, it is not difficult to observe that $x$ is the unique vertex of maximum degree with a monochromatic palette $\{1, \ldots, 1\}$. Thus, the whole subgraph $G^{\prime}$ (or $G_{+}^{\prime}$ ) is fixed.

To end the proof, we colour pendant trees and triangles deleted from $G$ at the beginning. First assume that $G^{\prime}$ is not empty. Let $N_{i}\left(G^{\prime}\right)$, for $i \geq 0$, be the set of vertices of distance $i$ from $G^{\prime}$. Then we recursively colour the edges incident to consecutive spheres $N_{i}\left(G^{\prime}\right)$ in the following way: for $v \in N_{i}\left(G^{\prime}\right), i \geq 0$, we colour all edges $v u$, where $u \in N_{i+1}\left(G^{\prime}\right)$, with distinct colours from $\{1, \ldots, \Delta-1\}$ and the remaining edges incident to $v$, contained in $N_{i}(x)$, with 2 . Hence, all vertices of $G$ will be fixed by any automorphism preserving our colouring $f$.

If $G^{\prime}$ is empty, then we start with the centre $Z(G)$ that is setwise fixed by every automorphism. It follows from Lemma 2.5 that $Z(G)$ either induces $K_{3}$, or $K_{2}$ (not contained in $K_{3}$ ), or $K_{1}$. Let first $Z(G)$ induce a triangle $K_{3}$. If $\Delta=3$, then we stabilize $Z(G)$ by colouring with two colours all edges incident with vertices of $Z(G)$. When $\Delta \geq 4$, we can colour the edges of the triangle $Z(G)$ with three colours. Next, we recursively colour edges incident to subsequent spheres $N_{i}(Z(G))$ with $\Delta-1$ colours.

If $Z(G)$ is an edge $e$, then $G-e$ has two components. We distinguish each of them 
by colouring subsequent spheres $N_{i}(Z(G))$ with $\Delta-1$ colours. If the components are isomorphic, then by assumption, each of them has a triangle. We colour two edges of these triangles contained in a sphere $N_{i}(Z(G))$, for some $i \geq 2$, with two distinct colours.

Finally, let $Z(G)$ be a single vertex $z$. Hence, $G-z$ has $q \geq 2$ components, each joined to $z$ by one or two edges. If $q<\Delta$, then we can easily colour distinguishingly the edges incident with subsequent spheres $N_{i}(z), i \geq 0$, with $\Delta-1$ colours. If $q=\Delta$, then we choose two components of $G-z$, at least one of them with a triangle, and colour their two edges incident with $z$ with the same colour. Then we distinguish these two components by an edge of the triangle.

\section{Some classes of graphs}

A graph $G$ is called asymmetric if its automorphism group is trivial. Then obviously $D^{\prime}(G)=1$.

We say that a graph $G$ is almost spanned by a subgraph $H$ (not necessarily connected) if $G-v$ is spanned by $H$ for some $v \in V(G)$. The following observation will play a crucial role in this section.

Lemma 3.1. If a graph $G$ is spanned or almost spanned by a subgraph $H$, then

$$
D^{\prime}(G) \leq D^{\prime}(H)+1 \text {. }
$$

Proof. We colour the edges of $H$ with colours $1, \ldots, D^{\prime}(H)$, and all other edges of $G$ with an additional colour 0 . If $\varphi$ is an automorphism of $G$ preserving this colouring, then $\varphi(x)=x$, for each $x \in V(H)$. Moreover, if $H$ is a spanning subgraph of $G-v$, then also $\varphi(v)=v$. Therefore, $\varphi$ is the identity.

\subsection{Traceable graphs}

Recall that a graph is traceable if it contains a Hamiltonian path.

Theorem 3.2. If $G$ is a traceable graph of order $n \geq 7$, then $D^{\prime}(G) \leq 2$.

Proof. Let $P_{n}=v_{1} v_{2} \ldots v_{n}$ be a Hamiltonian path of $G$. If $G=P_{n}$, then the conclusion follows from Proposition 2.1. If $G$ is isomorphic to $P_{n}+v_{1} v_{3}$, then we colour the edge $v_{1} v_{3}$ with 1 , and all other edges with 2 breaking all non-trivial automorphisms of $G$. So suppose that $G$ contains an edge $v_{i} v_{j}$ distinct from $v_{1} v_{3}$ and $v_{n-2} v_{n}$ with $i<j-1$. Without loss of generality we may assume that $i-1 \leq n-j$ (otherwise we reverse the labeling). It is easy to see that at least one of the graphs $P_{n}+v_{i} v_{j}-v_{j-1} v_{j}, P_{n}+v_{i} v_{j}-v_{j-1}$ or $P_{n}+v_{i} v_{j}-v_{n}$ is an asymmetric spanning or almost spanning subgraph of $G$ for any $n \geq 7$. The conclusion follows from Lemma 3.1.

The assumption $n \geq 7$ is substantial in Theorem 3.2 as $D^{\prime}\left(K_{3,3}\right)=3$.

\subsection{Claw-free graphs}

A $K_{1,3}$-free graph, called also a claw-free graph, is a graph containing no copy of $K_{1,3}$ as an induced subgraph. Claw-free graphs have numerous applications, e.g., in operations research and scheduling theory. For a survey of claw-free graphs and their applications consult [10].

A $k$-tree of a connected graph is its spanning tree with maximum degree at most $k$. Win [21] investigated spanning trees in 1-tough graphs and proved the following result. 
Theorem 3.3 ([21]). A 2-connected claw-free graph has a 3-tree.

We use this result to give an upper bound for the distinguishing number of claw-free graphs.

Theorem 3.4. If $G$ is a connected claw-free graph, then $D^{\prime}(G) \leq 3$.

Proof. Assume first that $G$ is 2-connected. By Theorem 3.3, $G$ contains a 3-tree $T$. By Theorem 2.6, we have $D^{\prime}(T) \leq 2$ if $T$ is neither symmetric nor bisymmetric tree. In such a case, $D^{\prime}(G) \leq 3$ by Lemma 3.1 .

Let $T$ be a symmetric tree $T_{h, 3}$. Denote a central vertex of $T$ by $x$ and its neighbours by $a, b, c$. Since $G$ is a claw-free graph, there exists in $G$ at least one edge, say $b c$, in the neighbourhood of $x$ in $T$. Define a subgraph $\widetilde{T}=T+b c$. We colour $b c, x a$ and $x b$ with 1 , and $x c$ with 2 . Thus all vertices $a, b, c, x$ are fixed by every non-trivial automorphism of $\widetilde{T}$. We now colour the remaining edges in $\widetilde{T}$ starting from the edges incident to $a, b, c$ in such a way that two uncoloured adjacent edges obtain two different colours 1 and 2 . This 2-colouring breaks all non-trivial automorphisms of $\widetilde{T}$. Hence, $D^{\prime}(G) \leq 3$ by Lemma 3.1.

Let $T$ be a bisymmetric tree $T_{h, 3}^{\prime \prime}$. Denote a central edge by $x y$ and its neighbours by $a, b$ adjacent to $x$, and $c, d$ adjacent to $y$. We colour $x y, x a$ and $y c$ with 1 , and $x b$ and $y d$ with 2. Since $G$ is claw-free, there exists in $G$ either at least one of the edges $b y, c x$ (or symmetrically $d x$ or $a y$ ) or both $a b$ and $c d$. We define a subgraph $\widetilde{T}$ obtained from the tree $T$ by adding either one of the edges $b y, c x$ (or symmetrically, $d x$ or $a y$ ) or both $a b$ and $c d$. In the first case we colour by or $c x$ (or symmetrically, $d x$ or $a y$ ) with 1 , in the second case we colour $a b$ with 1 and $c d$ with 2 . Now all vertices $a, b, c, d, x, y$ are fixed by every non-trivial automorphism of $\widetilde{T}$. We then colour the remaining edges of $\widetilde{T}$ as above, and we obtain the claim.

If a graph $G$ is not 2-connected, then its graph of blocks and cut-vertices is a path, since $G$ is claw-free. We colour every block according to the rules described above. Then to break all non-trivial automorphisms of $G$, it is enough to break a possible automorphism $\psi \in \operatorname{Aut}(G)$ that exchanges two terminal blocks. Let $z$ be a cut-vertex that belongs to a terminal block $B_{0}$. It follows that $z$ and its neighbours in $B_{0}$ induce a clique $K$ of order $k \geq 2$. We have three colours in our disposal, so it is easily seen that we can permute the colours to obtain a nonisomorphic colouring of $K$, thus breaking $\psi$.

The theorem is sharp for graphs of order at most 5 . We conjecture that the distinguishing index of claw-free graphs of order big enough is 2 .

\subsection{Planar graphs}

First, recall that by the famous Theorem of Tutte [19], every 4-connected planar graph $G$ is Hamiltonian. Hence, its distinguishing index is at most 2, by Theorem 3.2, whenever $|G| \geq 7$. A similar result as for claw-free graphs we obtain for 3-connected planar graphs. In the proof, we use the following result of Barnette about spanning trees of such graphs.

Theorem 3.5 ([3]). Every 3-connected planar graph has a 3-tree.

Using a similar method as in the proof of Theorem 3.4, we obtain the following.

Theorem 3.6. If $G$ is 3-connected planar graph, then $D^{\prime}(G) \leq 3$. 
Proof. Let $T$ be a 3-tree of $G$. It follows from Theorem 2.6 that $D^{\prime}(T) \leq 2$ and hence, $D^{\prime}(G) \leq 3$ by Lemma 3.1, if $T$ is neither a symmetric nor a bisymmetric tree.

Let then $T$ be a symmetric tree $T_{h, 3}$. Denote the central vertex by $x$, and by $T_{a}, T_{b}$ and $T_{c}$ the connected components of $T-x$ which are trees rooted at the neighbours $a, b, c$ of a vertex $x$, respectively. Since $G$ is 3 -connected, there exist an edge $e$ between $T_{a}$ and $T_{b}$ in $G$. Consider a spanning subgraph $\widetilde{T}=T+e$. Then we colour $x a$ and $x c$ with 1 , and $x b$ with 2 , and extend this colouring as in the proof of Theorem 3.4 to a colouring of $\widetilde{T}$ breaking all non-trivial automorphisms of $\widetilde{T}$ (the colour of $e$ is irrelevant). Consequently, $D^{\prime}(G) \leq 3$ by Lemma 3.1 .

If $T$ is a bisymmetric tree $T_{h, 3}^{\prime \prime}$ with the central edge $x y$, then we can add to $T$ one edge in a subtree of $T-x y$ rooted at $x$, and such a graph can be easily distinguished by two colours. Again, our claim follows from Lemma 3.1.

\subsection{2-connected graphs}

For a 2-connected planar graph $G$, the distinguishing index may attain $1+\lceil\sqrt{\Delta(G)}\rceil$ as it is shown by the complete bipartite graph $K_{2, q}$ with $q=r^{2}$ for a positive integer $r$. In this case, $D^{\prime}\left(K_{2, q}\right)=r+1$ as it follows from the result obtained independently by Fisher and Isaak [11] and by Imrich, Jerebic and Klavžar [14]. They proved the following theorem. Actually, they formulated it for the distinguishing number $D\left(K_{p} \square K_{q}\right)$ of the Cartesian product of complete graphs, but $D^{\prime}\left(K_{p, q}\right)=D\left(K_{p} \square K_{q}\right)$.

Theorem 3.7 ([11, 14]). Let $p, q$, $d$ be integers such that $d \geq 2$ and $(d-1)^{p}<q \leq d^{p}$. Then

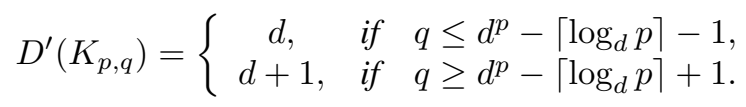

If $q=d^{p}-\left\lceil\log _{d} p\right\rceil$ then the distinguishing index $D^{\prime}\left(K_{p, q}\right)$ is either $d$ or $d+1$ and can be computed recursively in $O\left(\log ^{*}(q)\right)$ time.

In the next section, we make use of the following immediate corollary.

Corollary 3.8. If $p \leq q$, then $D^{\prime}\left(K_{p, q}\right) \leq\lceil\sqrt[p]{q}\rceil+1$.

In the proof of Proposition 3.10 we also make use of an earlier result of Imrich and Klavžar [15] which is a slightly weaker version of Theorem 3.7 for $d=2$.

Theorem 3.9 ([15]). If $2 \leq p \leq q \leq 2^{p}-p+1$, then $D^{\prime}\left(K_{p, q}\right)=2$.

Proposition 3.10. If $p \leq q \leq 2^{p}-p+1$ and $p+q \geq 7$, then there exists a distinguishing edge 2-colouring of $K_{p, q}$ such that the edges in one of colours induce a connected spanning or almost spanning, asymmetric subgraph of $K_{p, q}$.

Proof. The assumptions imply that $p \geq 3$, and $D^{\prime}\left(K_{p, q}\right)=2$ by Theorem 3.9. Let $P$ and $Q$ be the two sets of bipartition of $K_{p, q}$ with $|P|=p$ and $|Q|=q$. If $p=q$, then $p \geq 4$, and there exists a spanning asymmetric tree of $K_{p, p}$ (see [17]). If $p<q \leq 2^{p}-p+1$, then for the proof of Theorem 3.9, Imrich and Klavžar in [15] constructed a distinguishing vertex 2-colouring of $K_{p} \square K_{q}$ that corresponds to a distinguishing edge 2-colouring $f$ of $K_{p, q}$, where a colouring of vertices in a $K_{q}$-layer can be represented by a sequence from $\{1,2\}^{q}$ and it corresponds to a colouring of edges incident to a vertex in $P$ (the same is true 
for $K_{p}$-layers and vertices in $Q$ ). We wish to show that this colouring yields a connected asymmetric subgraph of $K_{p, q}$ which is spanning or almost spanning.

First assume that $q=2^{p}-p+1$. In the coloring $f$, every vertex in $P$ has distinct positive number of edges coloured with 1 , and there exists a vertex $v_{1}$ with all incident edges coloured with 1 . Moreover, distinct vertices from $Q$ have distinct sets of neighbours joined by edges coloured with 1 , and there exists a vertex, say $v_{2}$, with all incident edges coloured with 2 . Let $S$ be a subgraph induced by edges coloured with 1 . Then $S$ is an almost spanning subgraph since $v_{2}$ is the only vertex outside $S$. The graph $S$ is connected because $v_{1}$ is adjacent to every vertex in $Q$, and every vertex in $P$ is joined to a vertex in $Q$ by an edge coloured with 1 . Moreover, $S$ is also asymmetric since $f$ breaks all non-trivial automorphisms of $K_{p, q}$ and any automorphism interchanging some parts of the sets $P$ and $Q$ does not preserve distances in $S$.

Following [15] for $p<q<2^{p}-p+1$, we exclude a relevant number of such pairs of sequences of colours that the sum of them is a sequence $(3, \ldots, 3)$. Additionally, if both $q$ and $p$ are odd, we exclude the sequence $(0, \ldots, 0)$. Again, we obtain a connected spanning (or almost spanning) asymmetric subgraph $S$ of $K_{p, q}$ induced by the edges coloured with 1 .

Proposition 3.10 and Lemma 3.1 immediately imply the following.

Corollary 3.11. If a graph $G$ of order at least 7 is spanned by $K_{p, q}$ and $p \leq q \leq 2^{p}-p+1$, then $D^{\prime}(G) \leq 2$.

In general, for 2-connected graphs we conjecture that the complete bipartite graph $K_{2, r^{2}}$ is the worst case, i.e. attains the highest value of the distinguishing index.

Conjecture 3.12. If $G$ is a 2-connected graph, then

$$
D^{\prime}(G) \leq 1+\lceil\sqrt{\Delta(G)}\rceil
$$

\section{Nordhaus-Gaddum inequalities for $D^{\prime}$}

In this section, we discuss Conjecture 1.6, formulated at the end of Introduction, stating that

$$
2 \leq D^{\prime}(G)+D^{\prime}(\bar{G}) \leq \Delta+2
$$

for every admissible graph $G$ of order $n \geq 7$, where $\Delta=\max \{\Delta(G), \Delta(\bar{G})\}$.

The left-hand inequality is obvious. Indeed, if a graph $G$ is asymmetric, then so is $\bar{G}$. Thus we are only interested in the right-hand inequality $D^{\prime}(G)+D^{\prime}(\bar{G}) \leq \Delta+2$. Note also that at least one of the graphs $G$ and $\bar{G}$ is connected.

The bound $\Delta+2$ cannot be improved. To see this, consider a star $K_{1, n-1}$ of any order $n \geq 7$. As $\overline{K_{1, n-1}}$ is a disjoint union of a complete graph $K_{n-1}$ and an isolated vertex, it follows from Proposition 2.3 that $D^{\prime}\left(\overline{K_{1, n-1}}\right)=2$. Therefore, $D^{\prime}\left(K_{1, n-1}\right)+$ $D^{\prime}\left(\overline{K_{1, n-1}}\right)=n-1+2=\Delta+2$.

If $T$ is a tree, then $\Delta(T)$ can be much smaller than $\Delta=\Delta(\bar{T})=n-1$. However, the following holds.

Proposition 4.1. If $T$ is a tree of order $n \geq 7$, then

$$
D^{\prime}(T)+D^{\prime}(\bar{T}) \leq \Delta(T)+2 .
$$


Proof. As it was shown above, the conclusion holds for stars. If $T$ is not a star, then $D^{\prime}(\bar{T}) \leq 2$ by Lemma 3.1. Indeed, as it was proved by Hedetniemi et al. in [13], a complete graph $K_{n}$ contains edge disjoint copies of any two trees of order $n$ distinct from a star $K_{1, n-1}$. Thus, the complement $\bar{T}$ contains a spanning asymmetric tree. By Theorem 2.6, we have the inequality $D^{\prime}(T)+D^{\prime}(\bar{T}) \leq \Delta(T)+2$.

This fact emboldened us to formulate the following stronger conjecture.

Conjecture 4.2. Every connected admissible graph $G$ of order $n \geq 7$ satisfies the inequality

$$
D^{\prime}(G)+D^{\prime}(\bar{G}) \leq \Delta(G)+2 .
$$

Now we show that Conjecture 1.6 holds not only for trees, but also for some other classes of graphs. To do this we use the following fact.

Theorem 4.3. Let $G$ be a connected admissible graph of order $n \geq 7$. If either $G$ or every connected component of $\bar{G}$ has the distinguishing index at most 3 , then

$$
D^{\prime}(G)+D^{\prime}(\bar{G}) \leq \Delta+2
$$

where $\Delta=\max \{\Delta(G), \Delta(\bar{G})\}$.

Proof. Our claim is true for trees by Proposition 4.1. Observe also, that it is true if $G$ is a path or a cycle of order at least 7 since its complement $\bar{G}$ is Hamiltonian, and $D^{\prime}(G)+$ $D^{\prime}(\bar{G}) \leq 4$. So, now we can assume that $\Delta(G) \geq 3$ and neither $G$ nor $\bar{G}$ is a tree. We consider two cases.

Case A. Every component $H$ of $\bar{G}$ satisfies $D^{\prime}(H) \leq 3$.

Then $D^{\prime}(G) \leq \Delta(G)-1$ by Theorem 2.8 , and if $\bar{G}$ is connected, then our claim holds. Assume now that $\bar{G}$ is disconnected. Then $G$ is spanned by $K_{p, q}$ with $p \leq q$ and $\Delta \geq q$, where $p+q=|V(G)|$. Suppose that the graph $\bar{G}$ has $t$ isomorphic components. If we had a distinct set of three colours for every component, then $D^{\prime}(\bar{G}) \leq\lceil\sqrt[3]{6 t}\rceil$. We then consider two cases:

a) If $q \leq 2^{p}-p+1$, then $D^{\prime}(G)=2$ by Corollary 3.11. Moreover, we then have at most $\frac{n}{3}$ components of $\bar{G}$, so $D^{\prime}(\bar{G}) \leq\lceil\sqrt[3]{2 n}\rceil$. And we can easily see that

$$
\lceil\sqrt[3]{2 n}\rceil+2 \leq \frac{n}{2}+2
$$

for every $n \geq 4$.

b) If $q \geq 2^{p}-p+1$, then there exists a big component (of order $q$ ) in $\bar{G}$ and we can assume that $t \leq \frac{p}{3}$ remaining components are isomorphic. In this case, by assumptions we have $p \leq\left\lceil\log _{2}(q+p-1)\right\rceil$, therefore

$$
D^{\prime}(\bar{G}) \leq\lceil\sqrt[3]{6 t}\rceil \leq \sqrt[3]{2\left\lceil\log _{2}(q+p-1)\right\rceil} .
$$

On the other hand, $D^{\prime}(G) \leq\lceil\sqrt[p]{q}\rceil+2$ by Corollary 3.8 and Theorem 3.1. Then it is not difficult to check that for $q \geq 2^{p}-p+1$

$$
\sqrt[3]{2\left\lceil\log _{2}(q+p-1)\right\rceil}+\lceil\sqrt[p]{q}\rceil+2 \leq q+2
$$

what finishes the proof in Case A. 
Case B. $D^{\prime}(G) \leq 3$.

If graph $\bar{G}$ is connected, then the claim follows immediately from Theorem 2.7 whenever $D^{\prime}(G)=2$ or $D^{\prime}(\bar{G})=2$, and it follows from Theorem 2.8 if $D^{\prime}(G)=3$. Assume now that $\bar{G}$ has $t \geq 2$ components. Then $\Delta \geq \frac{n}{2}$ and, in the worst case, all components of $\bar{G}$ are isomorphic. Observe that maximal degree of every component is at most $\frac{n}{t}-1$. If we assign one extra colour to every component, then we need at most $\frac{n}{t}-1+(t-1)$ colours to distinguish $\bar{G}$. Hence, if

$$
\frac{n}{t}+t \leq \frac{n}{2}-1
$$

then $D^{\prime}(\bar{G}) \leq \Delta-1$, and our claim is true. The above inequality holds unless $t=2$.

If there exist two isomorphic components in $\bar{G}$, then $D^{\prime}(G) \leq 2$ due to Corollary 3.11 since $G$ is spanned by $K_{\frac{n}{2}, \frac{n}{2}}$. Then $D^{\prime}(\bar{G}) \leq \frac{n}{2}$, and finally $D^{\prime}(G)+D^{\prime}(\bar{G}) \leq \frac{n}{2}+2$.

Now we can formulate some consequences of Theorem 4.3 and suitable results proved in Section 3.

Corollary 4.4. Let $G$ be an admissible graph of order $n \geq 7$. If $G$ satisfies at least one of the following conditions:

i) G is a traceable graph, or

ii) $G$ is a claw-free graph, or

iii) $G$ is a triangle-free graph, or

iv) G is a 3-connected planar graph,

then

$$
D^{\prime}(G)+D^{\prime}(\bar{G}) \leq \Delta+2,
$$

where $\Delta=\max \{\Delta(G), \Delta(\bar{G})\}$.

Proof. It suffices to apply Theorem 4.3 together with Theorem 3.2, Theorem 3.4 and Theorem 3.6, respectively. Observe also that if the girth of a graph $G$ is at least 4, i.e., $G$ is triangle-free, then its complement $\bar{G}$ is claw-free.

Finally, it has to be noted that there exist graphs of order less than 7 such that the righthand inequality in Conjecture 1.6 is not satisfied. For example, for the graph $K_{3,3}$ we have $D^{\prime}\left(K_{3,3}\right)=3, D^{\prime}\left(\overline{K_{3,3}}\right)=D^{\prime}\left(2 K_{3}\right)=4$ and $\Delta=3$, hence $D^{\prime}\left(K_{3,3}\right)+D^{\prime}\left(\overline{K_{3,3}}\right)=$ $\Delta+4$. Also, $D^{\prime}\left(C_{5}\right)+D^{\prime}\left(\overline{C_{5}}\right)=3+3=\Delta+4$, and $D^{\prime}\left(K_{1, i}\right)+D^{\prime}\left(\overline{K_{1, i}}\right)=\Delta+3$ for $i=3,4,5$.

\section{References}

[1] M. O. Albertson and K. L. Collins, Symmetry breaking in graphs, Electron. J. Combin. 3 (1996), \#R18, http://www.combinatorics.org/ojs/index.php/eljc/ article/view/v3ilr18.

[2] M. Aouchiche and P. Hansen, A survey of Nordhaus-Gaddum type relations, Discrete Appl. Math. 161 (2013), 466-546, doi:10.1016/j.dam.2011.12.018.

[3] D. W. Barnette, Trees in polyhedral graphs, Canad. J. Math. 18 (1966), 731-736, doi:10.4153/ cjm-1966-073-4. 
[4] D. L. Boutin, The cost of 2-distinguishing Cartesian powers, Electron. J. Combin. 20 (2013), \#P74, http: / / www. combinatorics.org/ojs/index.php/eljc/ article/view/v20i1p74.

[5] J. O. Choi, S. G. Hartke and H. Kaul, Distinguishing chromatic number of Cartesian products of graphs, SIAM J. Discrete Math. 24 (2010), 82-100, doi:10.1137/060651392.

[6] K. L. Collins, M. Hovey and A. N. Trenk, Bounds on the distinguishing chromatic number, Electron. J. Combin. 16 (2009), \#R88, http: / www. combinatorics.org/ojs / index.php/eljc/article/view/v16ilr88.

[7] K. L. Collins and A. N. Trenk, The distinguishing chromatic number, Electron. J. Combin. 13 (2006), \#R16, http: / / www. combinatorics.org/ojs/index.php/eljc/ article/view/v13ilr16.

[8] K. L. Collins and A. N. Trenk, Nordhaus-Gaddum theorem for the distinguishing chromatic number, Electron. J. Combin. 20 (2013), \#P46, http://www.combinatorics.org/ ojs/index.php/eljc/article/view/v20i3p46.

[9] E. Estaji, W. Imrich, R. Kalinowski, M. Pilśniak and T. W. Tucker, Distinguishing Cartesian products of countable graphs, Discuss. Math. Graph Theory 37 (2017), 155-164, doi:10.7151/ dmgt.1902.

[10] R. Faudree, E. Flandrin and Z. Ryjáček, Claw-free graphs - a survey, Discrete Math. 164 (1997), 87-147, doi:10.1016/s0012-365x(96)00045-3.

[11] M. J. Fisher and G. Isaak, Distinguishing colorings of Cartesian products of complete graphs, Discrete Math. 308 (2008), 2240-2246, doi:10.1016/j.disc.2007.04.070.

[12] R. Hammack, W. Imrich and S. Klavžar, Handbook of Product Graphs, Discrete Mathematics and Its Applications, CRC Press, Boca Raton, Florida, 2nd edition, 2011, doi:10.1201/b10959.

[13] S. M. Hedetniemi, S. T. Hedetniemi and P. J. Slater, A note on packing two trees into $K_{n}$, Ars Combin. 11 (1981), 149-153.

[14] W. Imrich, J. Jerebic and S. Klavžar, The distinguishing number of Cartesian products of complete graphs, European J. Combin. 29 (2008), 922-929, doi:10.1016/j.ejc.2007.11.018.

[15] W. Imrich and S. Klavžar, Distinguishing Cartesian powers of graphs, J. Graph Theory 53 (2006), 250-260, doi:10.1002/jgt.20190.

[16] W. Imrich, S. M. Smith, T. W. Tucker and M. E. Watkins, Infinite motion and 2distinguishability of graphs and groups, J. Algebraic Combin. 41 (2015), 109-122, doi: 10.1007/s10801-014-0529-2.

[17] R. Kalinowski and M. Pilśniak, Distinguishing graphs by edge-colourings, European J. Combin. 45 (2015), 124-131, doi:10.1016/j.ejc.2014.11.003.

[18] E. A. Nordhaus and J. W. Gaddum, On complementary graphs, Amer. Math. Monthly 63 (1956), 175-177, doi:10.2307/2306658.

[19] W. T. Tutte, A theorem on planar graphs, Trans. Amer. Math. Soc. 82 (1956), 99-116, doi: $10.2307 / 1992980$.

[20] V. G. Vizing, The chromatic class of a multigraph, Kibernetika 1 (1965), 29-39, doi:10.1007/ bf01885700.

[21] S. Win, On a connection between the existence of $k$-trees and the toughness of a graph, Graphs Combin. 5 (1989), 201-205, doi:10.1007/bf01788671.

[22] A. A. Zykov, On some properties of linear complexes, Mat. Sbornik (N. S.) 24 (1949), 163-188, http://mi.mathnet.ru/eng/msb5974. 